\title{
Valor pronóstico del marcador de superficie CD56 en pacientes con mieloma múltiple
}

- Mónica Londoño, Humberto Martínez Cordero, Martha Suárez Acuña, Leonardo Enciso Olivera, Diana Gil Calderón, Claudia Agudelo López, Carmen Lucía Roa, Sandra Chinchilla, Fernanda Bedón, Andrea Naranjo

Instituto Nacional de Cancerología, Bogotá, D.C.

Contacto: humbertomartinez48@hotmail.com

\begin{abstract}
Introducción y objetivos. El mieloma múltiple es una enfermedad catastrófica con desenlaces altamente heterogéneos y con supervivencias que varían desde pocos meses hasta más de 10 años. La negatividad del clúster de diferenciación CD56 se ha correlacionado con pobres desenlaces en los pacientes con esta enfermedad. El objetivo del presente trabajo es determinar la correlación de este marcador con la estratificación de riesgo al diagnóstico y la supervivencia.
\end{abstract}

Materiales y métodos. Estudio de cohorte retrospectivo que incluye pacientes con diagnóstico de mieloma múltiple. Se verifica la clasificación de riesgo al diagnóstico, se determina la tasa de supervivencia y se correlaciona con la positividad o negatividad para el marcador de superficie CD56.

Resultados. Se incluyeron 86 pacientes. De estos, 39 pacientes fueron mujeres, la mediana de edad al diagnóstico fue de 57,58 años. Se encontraron 74 pacientes con CD56 positivo y 12 pacientes con CD56 negativo ( $86 \%$ y $14 \%$, respectivamente). No hubo diferencias estadísticamente significativas en cuanto a la estratificación en alto riesgo de la enfermedad al diagnóstico $(56,98 \%$ vs. $58,1 \%, p=0,23)$ ni en la mediana de supervivencia en un período de evaluación de seis años (66 semanas vs. 71 semanas, $p=0,10$ ).

Conclusiones. La negatividad del marcador de superficie CD56 no se correlaciona con la estratificación de alto riesgo al diagnóstico ni con peor supervivencia.

\section{Histiocitosis de células de Langerhans: experiencia en el Hospital de la Misericordia en 26 años}

\author{
- Agustín Contreras, Édgar Cabrera, Fabio Restrepo, Adriana Linares, Isabel Sarmiento, Carlos Pardo, Lina Jaramillo, \\ Jimmy Lagos, Francy Ortiz
}

Fundación Hospital de la Misericordia, Bogotá D.C.

Contacto: acontrerasa1@gmail.com

Introducción y objetivos. Objetivo: describir el curso clínico de la histiocitosis de células de Langerhans (HCL).

Materiales y métodos. Métodos: estudio descriptivo y retrospectivo de casos clínicos de menores de 18 años con diagnóstico de HCL en un centro de oncología pediátrica desde 1988 hasta 2014.

Resultados. Se encontraron 121 pacientes, con mediana de edad de 24 meses (rango 6-192), predominio de sexo femenino. Las lesiones óseas fueron la manifestación más frecuente de la enfermedad en 83 casos (68\%), seguida por compromiso en piel $(37 \%)$, médula ósea $(33,8 \%)$, bazo $(29,7 \%)$, hígado $(28,9 \%)$, ganglios cervicales (13\%), diabetes insípida (9\%), compromiso del canal auditivo y pulmón (8\% cada uno). Se encontró compromiso monosistémico en 51 casos (42\%) y multisistémico en 70 (58\%), de los cuales 58 pacientes (82\%) tenían compromiso de órgano de riesgo (hígado, bazo o MO), 12 pacientes (10\%) tuvieron reactivación de la enfermedad, 11 (9\%) abandonaron el tratamiento y 12 (10\%) fallecieron por progresión de la enfermedad. La sobrevida global a cinco años fue del $90 \%$.

Conclusiones. Se evidencia que los menores de un año tienen mayor compromiso multisistémico y con implicación de órgano de riesgo, lo que hizo que recibieran terapias más intensivas y seguimiento más estrecho, obteniendo buenos resultados, con una sobrevida del $68 \%$. Los mayores de 10 años tienen más compromiso monosistémico y mejor pronóstico. En general, las manifestaciones y el comportamiento clínico de la $\mathrm{HCL}$ en nuestros pacientes son iguales de amplios a los registrados en la literatura mundial. 